\section{Radiology for PET/CT Reporting}

C. Nanni, S. Fanti, and L. Zanoni, Eds.

New York, NY: Springer, 2014, 149 pages, $\$ 59.99$

PET/CT scans are of great value to oncologists, surgeons, and other medical specialists when the metabolic and anatomic information is properly integrated and the imaging findings are put into the context of the patient's specific medical history. These examinations, however, are among the most complex and time-consuming medical imaging studies to interpret and report. Not infrequently, abnormal findings on CT images are functionally silent and thus difficult for nuclear medicine physicians to interpret. Furthermore, in general only a low-dose CT scan is produced as part of the combined PET/CT study, and the resulting CT images may prove suboptimal for image interpretation.

The tasks of PET/CT examination begin with full interpretation of PET and CT images and integration of the findings. The PET/CT report should be treated as a consultation, with particular attention to a concise history and an impression that directly addresses the key clinical issues relevant to the patient's subsequent management. PET/CT reports require an added depth because of the complex treatment history and number of prior imaging studies for the patient. The incidental findings depicted on either PET or CT images must be recognized and reported. The impression should answer the question that the referring physician has posed.

This small atlas with 6 chapters is designed to enable nuclear medicine practitioners who routinely interpret PET/CT scans to recognize and report the most common $\mathrm{CT}$ abnormalities. All the images were obtained by the 3 editors in Bologna, Italy, and are unenhanced CT images. There are no PET or fused colorful PET/CT images. The first chapter deals with normal anatomy on low-dose CT images, identifying all the relevant anatomic structures that can help in accurately describing abnormalities seen on PET images. The other chapters, on the head and neck, thorax, abdomen, pelvis, and musculoskeletal system, present cases with common and uncommon ${ }^{18} \mathrm{~F}$-FDG-negative anatomic abnormalities. All images are clear and can be directly compared with the CT images acquired on a standard PET/CT scanner. There are no tables, references, or index. This book will be helpful to trainees and practitioners in the fields of nuclear medicine and technology.

\section{E. Edmund Kim University of California at Irvine 101 The City Dr. S. Orange, CA 92868 E-mail:edmundek@uci.edu}

Published online Dec. 4, 2014. DOI: 10.2967/jnumed.114.148692

\section{Nuclear Medicine: A Guide for Healthcare Professionals and Patients}

\author{
D. Prakash
}

New York, NY: Springer, 2014, 154 pages, $\$ 59.99$

During the modern era of radionuclide imaging and therapy, many nuclear medicine textbooks have been published in an attempt to educate not only practitioners of nuclear medicine but also referring physicians and medical students. Nuclear medicine procedures involve administration of radioactive material, and the optimal level of radioactivity should be used. Patients may have many apprehensions or questions during the procedures. More than 50 procedures are performed in the nuclear medicine service, and it may be difficult for nuclear medicine professionals to precisely remember everything they need to know about these procedures. The paramedical staff often sends patients for nuclear medicine procedures without proper preparation because the staff lacks knowledge about the subject. Many doctors are not familiar with nuclear medicine because they do not have enough time to learn about this medical specialty and thus are unable to use it for diagnostic and therapeutic purposes.

This relatively small book addresses all the issues mentioned above. It gives patients a general idea about particular investigations and therapies. It gives the paramedical staff members a concise education about a subject so that they can prepare patients adequately before sending them for a procedure and can build the patient's trust by being able to answer questions. It helps nuclear medicine professionals by serving as a ready reckoner to teach how the procedure is performed and the type and amount of radioactivity to be used. It gives referring physicians a broad overview of the subject by explaining procedures briefly and listing the common indications and precautions for each.

This book, by 4 contributors in India, is divided into 5 chapters. The first introduces nuclear medicine, including major types of equipment. The second describes the nuclear medicine team, comprising nuclear medicine physician, physicist, radiation safety officer, and personal physician. The third discusses common apprehensions about nuclear medicine, and the fourth covers nuclear medicine procedures. The descriptions of the procedures, based on guidelines of the Society of Nuclear Medicine and Molecular Imaging, the European Association of Nuclear Medicine, the American College of Radiology, and the International Atomic Energy Agency, are simple, easy to understand, and current. The last chapter defines common terminology used in nuclear medicine. The illustrative images are clear, the tables helpful, and the references current. There is no index. This book should be useful to nuclear medicine professionals, the paramedical staff, health care professionals, and general or referring physicians.

\author{
E. Edmund Kim \\ University of California at Irvine \\ 101 The City Dr. S. \\ Orange, CA 92868 \\ E-mail: edmundek@uci.edu
}

COPYRIGHT (c) 2015 by the Society of Nuclear Medicine and Molecular Imaging, Inc.
Published online Dec. 4, 2014.

DOI: 10.2967/jnumed.114.148809 